\title{
Dissipation in Highly Anisotropic Superconductors
}

\author{
P. H. Kes, J. Aarts, V. M. Vinokur, ${ }^{(a)}$ and C. J. van der Beek \\ Kamerlingh Onnes Laboratory, Leiden University, P.O. Box 9506, 2300 RA Leiden, The Netherlands
}

(Received 9 November 1989)

\begin{abstract}
In layered superconductors with very weak coupling between the layers the concept of a flux-line lattice breaks down when the field is oriented parallel to the superconducting planes. For an arbitrary field orientation we propose that the formation of an Abrikosov lattice is only related to the perpendicular field component. The parallel field component penetrates as if the superconducting planes were completely decoupled. This model explains recent experiments which have questioned the driving mechanism for dissipation in the superconducting phase of the high-temperature oxide superconductors.
\end{abstract}

PACS numbers: $74.60 . \mathrm{Ge}, 74.70 . \mathrm{Ya}$

Recently, serious questions have been raised about flux creep as the origin of dissipation in high-temperature superconductors. Iye, Nakamura, and Tamegai ${ }^{1}$ reported measurements of the resistivity $\rho$ in thin films of $\mathrm{Bi}_{2} \mathrm{Sr}_{2} \mathrm{CaCu}_{2} \mathrm{O}_{8}(\mathrm{Bi}: 2212)$ at temperatures down to $15 \mathrm{~K}$ below $T_{c}(=79 \mathrm{~K})$. When both the magnetic field $H$ and the current $I$ were oriented along the $\mathrm{CuO}_{2}$ planes, $\rho$ did not change with the variation of the angle $\phi$ between $H$ and $I$. Because the macroscopic driving force of a uniform current with density $J$ on a flux-line lattice (FLL) with flux density $B$ is given by $\mathbf{F}_{d}=\mathbf{J} \times \mathbf{B}$, one would expect $F_{d}$, to vary like $\sin \phi$, and therefore $\rho \propto \sin \phi$, if thermally activated flux flow ${ }^{2}$ is the origin of the dissipation. In contrast, similar experiments by Palstra et al. ${ }^{3}$ on single crystals of $\mathrm{YBa}_{2} \mathrm{Cu}_{3} \mathrm{O}_{7}(\mathrm{Y}: 123)$ showed a distinct resistance anisotropy for the cases $\phi=0$ and $\phi=\pi / 2$.

In this Letter, we propose a solution for the different observations in Refs. 1 and 3. The obvious conclusion from these experiments is that the resistance anisotropy in $\phi$ disappears for the material which exhibits the largest anisotropy of the superconducting parameters. Such anisotropy of the superconducting state is expressed as the ratio of the effective masses of the quasiparticles for motion in the $\mathrm{CuO}_{2}$ planes to those for motion normal to these planes, $m$ and $m_{z}$, respectively, and is defined by a parameter $\Gamma=m_{z} / m$. In a recent Letter by Farrell et al. ${ }^{4}$ describing transverse magnetization measurements on a $\mathrm{Bi}: 2212$ single crystal, a very large value of $\Gamma=3000$ was reported, whereas for $Y: 123$ it is $26 .^{5}$

This very large $\Gamma$ value has important consequences for the situation encountered when the field is exactly parallel to the $\mathrm{CuO}_{2}$ planes $(H \perp c)$. According to the Ginzburg-Landau (GL) theory for anisotropic superconductors, the field would penetrate in the form of a FLL consisting of isosceles triangles. ${ }^{6}$ For $B=1 \mathrm{~T}$ the size of the FLL unit cell in the $c$ direction would be $6.3 \mathrm{~nm}$ and $300 \mathrm{~nm}$ in the direction perpendicular to $c{ }^{4}$ Accepting that the flux-line cores are located between the superconducting $\mathrm{CuO}_{2}$ planes, ${ }^{7,8}$ the supercurrents circulating around the cores have to flow in the $\mathrm{CuO}_{2}$ planes and tunnel between them. For $B=20 \mathrm{~T}$, still not extremely high in view of the reported value of $45 \mathrm{~T} / \mathrm{K}$ for the slope of $B_{c 2}$ at $T_{c},{ }^{9}$ the distance between the FLL planes measured along $c$ would have decreased $\left(\propto B^{-1 / 2}\right)$ to 1.4 $\mathrm{nm}$, which is comparable to the distance between the $\mathrm{CuO}_{2}$ planes. At still higher fields the theory for anisotropic superconductors would predict more than one FLL plane between adjacent $\mathrm{CuO}_{2}$ planes. Consequently, the supercurrent pattern would not be identical for all vortices, which is in conflict with the conventional picture of a FLL.

The above "breakdown" of the Abrikosov lattice has not been previously considered, although it is related to the properties of layered superconductors which were studied in several papers. ${ }^{10-12}$ When the coupling between the superconducting layers is very weak, a description in terms of superconducting layers coupled by 2D Josephson junctions is more realistic than an anisotropic 3D Ginzburg-Landau model. In the 2D description the order parameter is large in the $\mathrm{CuO}_{2}$ planes, but almost uniformly zero between the layers. The field parallel to the planes penetrates in the form of Josephson vortices with mutual distance $a \approx \phi_{0} / B s$, where $s$ is the distance between the planes. (Note that the theory of 2D Josepshson junctions has to be revised for the oxide superconductors, because the superconductivity is located in a layer of thickness $0.3 \mathrm{~nm} .{ }^{13}$ ) Since the screening is very weak, both $H_{c 1}$ and the magnetization are extremely small $\left(<10^{-5} \mathrm{~T}\right.$ at $\left.T=0 \mathrm{~K}\right)$, i.e., the field penetrates between the decoupled superconducting layers as if the material is "magnetically transparent." The condition for the crossover from $3 \mathrm{D}$ to $2 \mathrm{D}$ is given by ${ }^{10}$

$$
\Gamma\left(1-t_{\mathrm{co}}\right)=2\left[\xi_{a b}(0) / s\right]^{2},
$$

where $t_{\mathrm{co}}=T_{\mathrm{co}} / T_{c}$ is the crossover temperature, and $\xi_{a b}$ is the Ginzburg-Landau coherence length in the $\mathrm{CuO}_{2}$ planes. For Bi:2212 with $\xi_{a b}(0)=3.2-3.8 \mathrm{~nm},{ }^{9,14} \mathrm{~s}=1.2$ $\mathrm{nm}$, and $\Gamma=3000$ we obtain $T_{c}-T_{\text {co }}=0.4-0.6 \mathrm{~K}$. For $\mathrm{Y}: 123$ with $\xi_{a b}(0)=2.0 \mathrm{~nm}, s=0.83 \mathrm{~nm}$, and $\Gamma=26$, we get $1-t_{\text {co }}=0.45$. So, according to this criterion, Y:123 should in practice always behave like an anisotropic 3D superconductor. In fact, a vortex lattice has been observed by Dolan et al. ${ }^{15}$ in Y:123 single crystals for fields 
both parallel and perpendicular to $c$. On the other hand, Bi:2212 will show 2D behavior half a kelvin below $T_{c}$.

Experimental evidence for the dimensionality of the superconductivity in thin films usually is obtained from the temperature and angular dependence of the upper critical field, or from the temperature dependence of the paraconductivity due to superconducting fluctuations. A difficulty with the high-temperature superconductors is that the transition field cannot be accurately determined from the resistive transition in a field, due to thermally activated flux flow. ${ }^{16}$ Nevertheless, a clear tendency is observed in the angular dependence with respect to the $\mathrm{CuO}_{2}$ planes. If $\theta$ is the angle between the field orientation and the normal to the planes, Naughton et al. ${ }^{14}$ find for $\mathrm{Y}: 123$ a smooth behavior at $H_{c 2}(\theta)$ around $\theta=\pi / 2$ in accord with the anisotropic 3D theory, whereas in Bi:2212 (Ref. 14) the 2D cuspedlike behavior as predicted by Tinkham ${ }^{17}$ is observed. The latter was also reported by Juang et al. ${ }^{18}$ Direct determination of $H_{c 2}$ from the reversible magnetization of a Y:123 single crystal ${ }^{19}$ confirms the results of Ref. 14.

Similar behavior of the resistively measured transition fields of single crystals of $\mathrm{Y}: 123$ with $\mathrm{O}_{6.9}$ and $\mathrm{O}_{6.6}$ has been very recently reported by Bauhofer et al. ${ }^{20} \mathrm{~A} 3 \mathrm{D}$ character is observed in the $\mathrm{O}_{6.9}$, while the $2 \mathrm{D}$ cusp is seen in the $\mathrm{O}_{6.6}$. Most striking is that the anisotropy of the latter composition is found to be $\Gamma=1600$. Furthermore these authors also see evidence for a dimensional crossover. Below $T_{\text {co }}$ which lies about $3 \mathrm{~K}$ below $T_{c}$ $(=58 \mathrm{~K})$, the critical field rises steeply. This would be in agreement with the $(1-t)^{1 / 2}$ dependence following from the 2D expression $H_{c \|}=2 \sqrt{6} H_{c} \lambda / d$, with $d$ the thickness of the $\mathrm{CuO}_{2}$ planes. Note that this expression yields $H_{c \|}(0)=2.5 \mathrm{kT}$, if the paramagnetic limiting is ignored, and that $J_{c}$ would be equal to the depairing current.

Regarding the superconducting fluctuations, recent results of Kim et al. ${ }^{21}$ show evidence for 2D fluctuations in thin films of $\mathrm{Tl}: 2212$ which is probably more anisotropic than Bi:2212. Earlier work of Oh et al. ${ }^{22}$ on films of Y:123 revealed evidence for a dimensional crossover. Both observations are consistent with the angular dependence of $H_{c 2}$. Additional evidence for the 2D behavior of $\mathrm{Bi}: 2212$ has been obtained by Martin et al. ${ }^{23}$ from the anisotropy of the zero-field critical-current densities.

From the above discussion it follows that Bi:2212 actually behaves as if the $\mathrm{CuO}_{2}$ planes are decoupled, i.e., the field parallel to the $\mathrm{CuO}_{2}$ planes penetrates completely. For arbitrary field orientations we now propose that only the perpendicular component $H_{\perp}$ gives rise to a vortex lattice with both the order-parameter zeros and the screening currents in the $\mathrm{CuO}_{2}$ layers. In the following we will first discuss how this model resolves the important questions raised in Ref. 1, and subsequently show that it is consistent with the results of resistance, magnetization, and torque measurements.

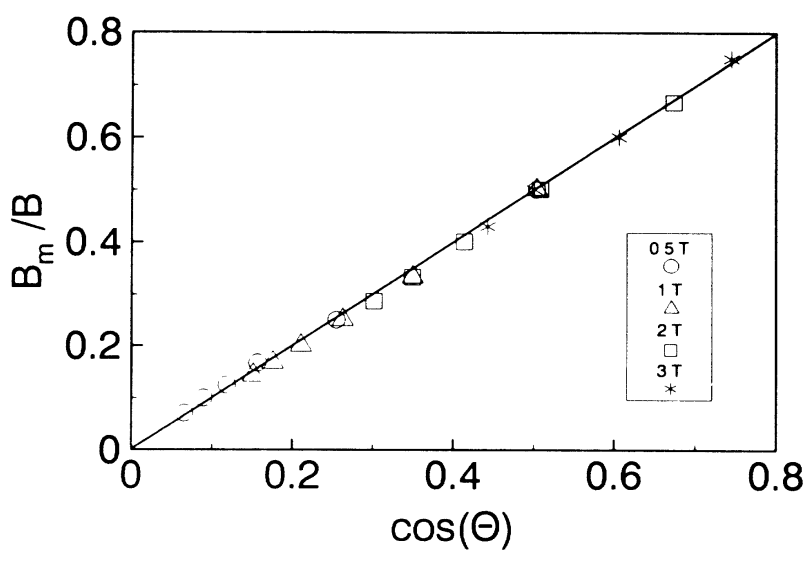

FIG. $1 . B_{m} / B$ vs $\cos \theta$ as determined from Fig. 2 of Ref. 1 displaying the fact that $\rho$ is constant for the same $B_{\perp}$.

For Iye, Nakamura, and Tamegai's experiment ${ }^{1}$ the consequences of the 2D model are clear. When $H \perp c$ there are no vortices and there should be no dissipation related to flux flow. However, considering the narrow angle tolerance set by the ratio of the sample or grain size and $s$, a perpendicular field component will inevitably occur, especially if the sample is not a perfect single crystal. Suppose $H_{\perp}=H_{0}$, then the minimum resistivities $\rho(T)$ as given in Fig. 6 of Ref. 1 should coincide with $\rho(T)$ data measured in a field $H_{0}$ directed perpendicular to the film. Such data are not given in Ref. 1, so that we cannot check this prediction. On the other hand, the data shown in Fig. 2 of Ref. 1, displaying $\rho$ as a function of $\theta$ (Ref. 24) for several applied fields can be nicely explained by our model. The dissipation is created by flux flow, because at the experimental conditions for $B$ and $T$ the FLL is thermally depinned. Applying a transport current with density $J$, therefore, immediately gives rise to flux flow according to $\rho \propto B_{\perp}$. Lines of constant $\rho$ drawn through the maxima at $\theta=0$ should cross the other curves at angles which obey the condition $\cos \theta=B_{m} / B$, where $B_{m}=B(\theta=0)$. In Fig. 1 we show several plots of $B_{m} / B$ vs $\cos \theta$ as obtained from the data of Ref. 1. The linear behavior is indeed observed with slope of 1 .

Other experimental results can be explained in a similar manner. We suggest that the resistivity measured by Palstra et al. ${ }^{3}$ in a Bi:2212 single crystal for $H \perp c$ is caused by a field component in the $c$ direction, possibly due to a small misalignment. Misalignments are also thought to be responsible for the observations of irreversible magnetization curves for $H \perp c .{ }^{25}$ In order to test this we show in Fig. 2 the results of a reinterpretation of the $J_{c}$ data obtained in Ref. 25 by dividing the difference in magnetization $\Delta M$ by $\cos \theta$ and multiplying $H$ with the same factor. Note that there is now only one relevant sample dimension which should be used in the Bean analysis. The best interpolation in Fig. 2 was obtained 


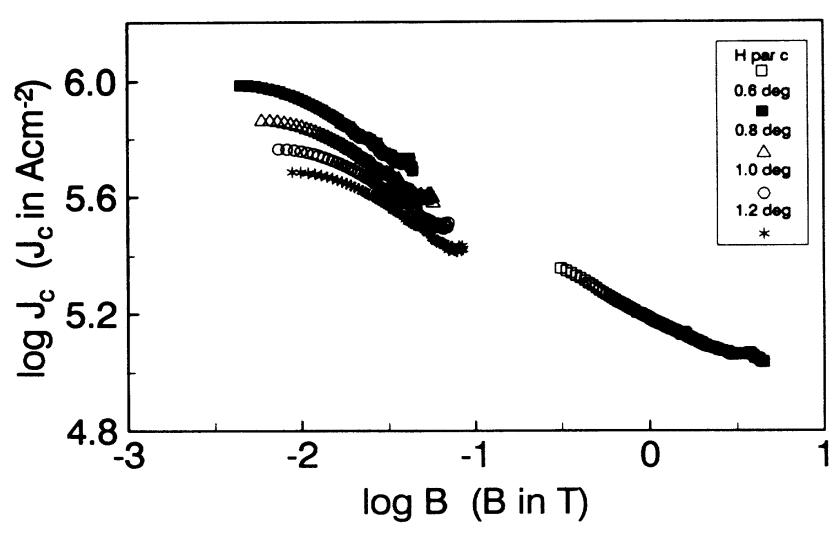

FIG. 2. Reinterpreted $J_{c}(B)$ data for a Bi:2212 single crystal as determined from magnetization measurements in field both parallel (open squares) and perpendicular to the $c$ axis for four misalignment angles (deviations from $\theta=\pi / 2$ ) as given in the figure.

with a misalignment of $0.8^{\circ}$.

The torque for the $2 \mathrm{D}$ case is simply

$$
\tau(\theta)=\mu_{0} M\left(H_{\perp}\right) V H \sin \theta,
$$

where $H_{\perp}=H \cos \theta$ and $V$ is the sample volume. For $H_{c 1} \ll H_{\perp} \ll H_{c 2}$ we use the 3D GL description for the magnetization, since a theory for a vortex lattice in a Josephson-coupled layered superconductor is not yet available. We then have ${ }^{26}$

$$
M=\left(H_{c 2, \perp} / 4 \kappa^{2}\right) \ln \left(\eta H_{c 2, \perp} / H_{\perp}\right) .
$$

Here $H_{c 2, \perp}$ is the upper critical field for $H \|_{c}, \eta$ is a constant of order unity, and $\kappa$ the GL parameter. When $H_{\perp}$ approaches $H_{c 1}$, for $\theta$ very close to $\pi / 2$, Eq. (3) is no longer valid, while $-M \propto H_{\perp}$ for $H_{\perp} \rightarrow 0$. We mimic this behavior by the interpolation formula $M^{\prime}$ $\left.=M\left(H_{\perp}\right) H_{\perp} /\left[M\left(H_{\perp}\right)+H_{\perp}\right)\right]$ with $M\left(H_{\perp}\right)$ given by Eq. (3). Using $M^{\prime}$ instead of $M$ in Eq. (2) we find that the torque measurements on $\mathrm{Bi}: 2212$ performed at 77.5 $\mathrm{K}$ as reported in Ref. 4 can be well described over the whole angular range by choosing $\kappa=60$ and $\eta H_{c 2, \perp}=18$. This is shown in Fig. 3. Using the value for $-d B_{c 2, \perp} / d T$ of $0.75 \mathrm{~T} / \mathrm{K},{ }^{9}$ we find $\eta=3.2$ and $H_{c 1}(77.5 \mathrm{~K})=3.2 \mathrm{mT}$, which are quite reasonable numbers. Finally, the assumed validity of the $2 \mathrm{D}$ model automatically invalidates the meaning of $\Gamma$; large values of $\Gamma$ are merely indicative for extremely large anisotropies.

The 2D behavior has important consequences for applications, as $J_{c}$ will be determined by $H_{\perp}$ only. Therefore $J_{c}$ for $\theta=\pi / 2$ can be much larger than for $\theta=0$; see Fig. 2. On the other hand, it was shown ${ }^{27,28}$ that a large $\Gamma$ gives rise to both strong fluctuation effects and very small energy barriers for thermally assisted depinning $U_{p}$ which will severely limit the application possibilities at high temperatures. For this purpose an enhancement of $U_{p}$ is required, preferably by improving the coupling and

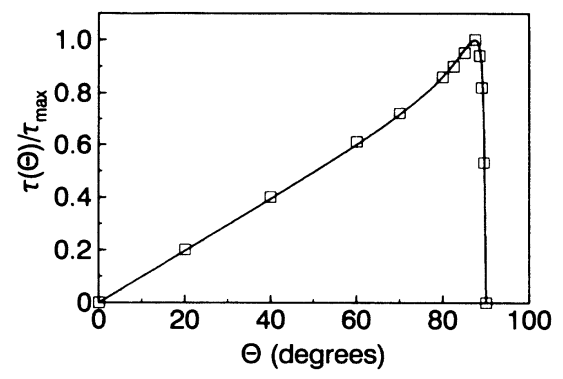

FIG. 3. Points selected from the normalized torque data of Ref. 4 on Bi:2212 at $B=1$ and $T=77.5 \mathrm{~K}$ (squares) compared with the prediction of the 2D theory (solid line): Eq. (2) using $M^{\prime}$ (see text).

reducing $\Gamma$.

Quite recently, Woo et al. ${ }^{29}$ addressed the issue raised in Ref. 1 in connection to experiments on Tl:2212 films. Gray, Kampwirth, and Farrell ${ }^{30}$ carried out torque measurements on similar films and observed a $\Gamma$ of about $10^{4}$. Clearly, the explanation given above for $\mathrm{Bi}: 2212$ holds equally well for the Tl films.

In summary, we propose that in very anisotropic superconductors in an applied field parallel to the superconducting layers the usual 3D magnetic behavior breaks down. From the dimensional-crossover criterion it is clear why Y:123 behaves three dimensionally and $\mathrm{Bi}: 2212$ two dimensionally. As a consequence of the 2D behavior the material is transparent for a magnetic field component parallel to the superconducting layers. The order parameter is finite in the layers and practically zero in between. This can be checked most sensitively by scanning-tunneling experiments ${ }^{31}$ on a $\mathrm{Bi}: 2212$ single crystal. The dissipation observed in resistance measurements on $\mathrm{Bi}: 2212$ well in the superconducting phase is due to thermally activated flux flow of the FLL created by the field component normal to the layers. In small fields and close to $T_{c}$ a Kosterlitz-Thouless transition can be observed. ${ }^{32,33}$ Important consequences can be expected for applications. Finally, it should be mentioned that these remarks also apply to artificially fabricated superconductor-insulator multilayers.

We thank J. van den Berg, A. J. Dirkmaat, and J. A. Mydosh for discussions and assistance during the preparation of this manuscript, and $\mathrm{W}$. Bauhofer and $\mathrm{K}$. E. Gray for sending preprints of their work. Part of this work has been supported by the Dutch Foundation for Fundamental Research on Matter (FOM).

\footnotetext{
(a) Permanent address: Institute of Solid State Physics, Chernogolovka, U.S.S.R.

${ }^{1} Y$. Iye, S. Nakamura, and T. Tamegai, Physica (Amsterdam) 159C, 433 (1989).

${ }^{2}$ P. H. Kes, J. Aarts, J. van den Berg, C. J. van der Beek, and J. A. Mydosh, Supercond. Sci. Technol. 1, 242 (1989).

${ }^{3}$ T. T. M. Palstra, B. Batlogg, L. F. Schneemeyer, and J. V.
} 
Waszczak, Phys. Rev. Lett. 61, 1662 (1988); T. T. M. Palstra, B. Batlogg, R. B. van Dover, L. F. Schneemeyer, and J. V. Waszczak, Appl. Phys. Lett. 54, 763 (1989).

${ }^{4}$ D. E. Farrell, S. Bonham, J. Foster, Y. C. Chang, P. Z. Jiang, K. G. Vandervoort, D. J. Lam, and V. G. Kogan, Phys. Rev. Lett. 63, 782 (1989).

${ }^{5}$ D. E. Farrell, C. M. Williams, S. A. Wolf, N. P. Bansal, and V. G. Kogan, Phys. Rev. Lett. 61, 2805 (1988).

${ }^{6}$ P. H. Kes, Physica (Amsterdam) 153-155C, 1121 (1988), and references cited therein; L. J. Campbell, M. M. Doria, and V. G. Kogan, Phys. Rev. B 38, 2439 (1988).

${ }^{7}$ D. Pines (private communication).

${ }^{8}$ M. Tachiki and S. Takahashi, Solid State Commun. 70, 291 (1989).

${ }^{9}$ T. T. M. Palstra, B. Batlogg, L. F. Schneemeyer, R. B. van Dover, and J. V. Waszczak, Phys. Rev. B 38, 5102 (1988).

${ }^{10} \mathrm{~W}$. E. Lawrence and S. Doniach, in Proceedings of the Twelfth International Conference on Low Temperature Physics, Kyoto, 1970, edited by E. Kanda (Keigaku, Tokyo, 1971), p. 361 .

${ }^{11}$ L. N. Bulaesvkii, Zh. Eksp. Teor. Fiz. 64, 2241 (1973); 94, 355 (1988) [Sov. Phys. JETP 37, 1133 (1973); 68, 1499 (1988)].

${ }^{12}$ R. A. Klemm, A. Luther, and M. R. Beasley, Phys. Rev. B 12, 877 (1975).

${ }^{13}$ A. A. Abrikosov, Fundamentals of the Theory of Metals (North-Holland, Amsterdam, 1988), p. 552.

${ }^{14}$ M. J. Naughton, R. C. Yu, P. K. Davies, J. E. Fischer, R. V. Chamberlin, Z. Z. Wang, T. W. Jing, N. P. Ong, and P. M. Chaikin, Phys. Rev. B 38, 9280 (1988).

${ }^{15}$ G. J. Dolan, F. Holtzberg, C. Feild, and T. R. Dinger, Phys. Rev. Lett. 62, 827 (1989); 62, 2184 (1989).

${ }^{16}$ A. P. Malozemoff, T. K. Worthington, Y. Yeshurun, F. Holtzberg, and P. H. Kes, Phys. Rev. B 38, 7203 (1989).

${ }^{17}$ M. Tinkham, Phys. Rev. 129, 2413 (1964).
${ }^{18}$ J. Y. Juang, J. A. Cutro, D. A. Rudman, R. B. van Dover, L. F. Schneemeyer, and J. V. Waszczak, Phys. Rev. B 38, 7045 (1988).

${ }^{19}$ U. Welp, W. K. Kwok, G. W. Crabtree, K. G. Vandervoort, and J. Z. Liu, Phys. Rev. Lett. 62, 1908 (1989).

${ }^{20}$ W. Bauhofer, W. Biberacher, B. Gegenheimer, W. Joss, R. K. Kremer, Hj. Mattausch, A. Müller, and A. Simon, Phys. Rev. Lett. 63, 2520 (1989).

${ }^{21}$ D. H. Kim, A. M. Goldman, J. H. Kang, K. E. Gray, and R. T. Kampwirth, Phys. Rev. B 39, 12275 (1989).

${ }^{22}$ B. Oh, K. Char, M. Naito, M. R. Beasley, T. H. Geballe, R. H. Hammond, A. Kapitulnik, and M. Graybeal, Phys. Rev. B 37, 7861 (1988).

${ }^{23}$ S. Martin, A. T. Fiory, R. M. Fleming, G. P. Espinosa, and A. S. Cooper, Appl. Phys. Lett. 54, 72 (1989).

${ }^{24}$ Note the different definitions of $\theta$ and $\phi$ in Ref. 1.

${ }^{25}$ J. van den Berg, C. J. van der Beek, P. H. Kes, J. A. Mydosh, M. J. V. Menken, and A. A. Menovsky, Supercond. Sci. Technol. 1, 249 (1989).

${ }^{26}$ P. G. de Gennes, Superconductivity of Metals and Alloys (Benjamin, New York, 1966), p. 71.

${ }^{27}$ L. J. de Jongh, Solid State Commun. 70, 955 (1989).

${ }^{28} \mathrm{P}$. H. Kes and J. van den Berg, in "Studies of High Temperature Superconductors," edited by A. Narlikar (Nova Science Publishers, New York, to be published).

${ }^{29}$ K. C. Woo, K. E. Gray, R. T. Kampwirth, J. H. Kang, S. J. Stein, R. East, and D. M. McKay, Phys. Rev. Lett. 63, 1877 (1989).

${ }^{30}$ K. E. Gray, R. T. Kampwirth, and D. E. Farrell (unpublished).

${ }^{31}$ H. F. Hess, R. B. Robinson, R. C. Dynes, J. M. Vales, and J. V. Waszczak, Phys. Rev. Lett. 62, 214 (1989).

${ }^{32}$ S. Martin, A. T. Fiory, R. M. Fleming, G. P. Espinosa, and A. S. Cooper, Phys. Rev. Lett. 62, 677 (1989).

${ }^{33}$ P. Minnhagen, Solid State Commun. 71, 25 (1989). 